\title{
Utilizing Supernova Remnants as Probes of Explosion Mechanisms and Progenitor Systems
}

\author{
Dan Milisavljevic \\ Harvard-Smithsonian Center for Astrophysics, 60 Garden Street, Cambridge, MA, 02138 \\ email: dmilisav@cfa.harvard.edu
}

\begin{abstract}
Theory and observation strongly favor the notion that asymmetric explosions drive core-collapse supernovae, but where and how this asymmetry becomes introduced is uncertain. The most likely places of origin are in the explosion mechanism itself or the interior structure of the star into which the explosion proceeds. Investigating the recently uncovered bubble-like interiors of young, nearby supernova remnants may provide a way to unravel which of these competing processes dominate.
\end{abstract}

Keywords. supernovae: general, supernova remnnats

Theory and observation strongly favor the notion that asymmetric explosions drive core-collapse supernovae ( $\mathrm{SNe}$ ). Where and how this asymmetry is introduced is uncertain, in part because of limited constraints on the various processes that may be taking place deep inside their massive progenitor stars. Some explosion asymmetries are potentially introduced by dynamical instabilities and the influences of rotation and magnetic fields, while others may be introduced by a perturbed progenitor star structure.

Observations of extragalactic SNe have provided some insight on this issue (e.g., Maeda et al. 2008). However, distant SNe by nature appear as unresolved point sources, which severely restricts the ability to obtain detailed knowledge of the three-dimensional kinematic and chemical properties of the expanding ejecta that can be used to extract key properties of the explosion dynamics.

One way around this problem is to examine young, nearby supernova remnants that encode valuable information about the core-collapse process in their debris. Cassiopeia A (Cas A) is one of the best specimens for post-mortem examination. Cas A shows many striking similarities with supernovae (e.g., SN 1993J; Milisavljevic et al. 2012) and other supernova remnants (e.g., 1E 0102-7219; Finkelstein et al. 2006; Milisavljevic et al., in preparation), and thus its dynamical properties can be used to help interpret other SN explosions and remnants that cannot be resolved.

A near-infrared spectroscopic survey of Cassiopeia A's interior unshocked S-rich material recently uncovered that the debris has a bubble-like morphology (Milisavljevic \& Fesen 2015). This large-scale "Swiss cheese-like" structure was most likely created early in the explosion from plumes of radioactive ${ }^{56} \mathrm{Ni}$-rich ejecta that rose up through outer shells of lighter elements. Such nickel-rich material would have decayed into cobalt and iron, releasing energy that would cause the gas to expand and compress nearby nonradioactive material such as oxygen, sulfur, and argon into cavity walls.

The amount of Ni produced and its distribution in a supernova are fundamental properties of the explosion that inform about the progenitor star's interior structure and the explosion mechanism. Interestingly, state-of-the-art computer simulations of corecollapse explosions attempting to incorporate asymmetries in the explosion mechanism 
and progenitor structure routinely find that the bulk of the Ni mass should remain inside the remnant with velocities below $2000 \mathrm{~km} \mathrm{~s}^{-1}$ (Hammer et al. 2010; Ono et al. 2013). However, this is actually opposite to what we currently see in Cas A, where X-ray-bright Fe emission traces the original Ni distribution out to velocities of $4000 \mathrm{~km} \mathrm{~s}^{-1}$ (DeLaney et al. 2010). Presently, there is no accepted understanding as to why this contradiction should be. Either the simulations are not adequately following the dynamics of mixing and/or more Fe remains to be detected in Cas A's interior.

Part of the problem may be rooted in the fact that explosion simulations use one dimensional progenitor star models. Recent observations have established that massive stars can undergo substantial and/or eruptive mass loss months to years before core collapse (Pastorello et al. 2013; Margutti et al. 2014), and present models are not incorporating the extensive non-spherical inter-shell mixing that must be occuring in the evolution of massive stars toward the ends of their life cycles (Smith \& Arnett 2014). If strong enough, pre-supernova dynamical interactions in the stellar interior may lead to Rayleigh-Taylor instabilities that can contribute to the formation of Ni-rich bubbles and influence the overall progression of the explosion.

Unraveling whether the asymmetries that we see in supernova remnants originate from asymmetric explosion mechanisms or the perturbed interior structure of the progenitor star is challenging but feasible. Progess can be made by (1) investigating the number and relative sizes of interior bubbles, and (2) determining the distributions of iron robustly.

\section{Acknowledgement}

This work was supported by NASA through grant GO-13378 from the Space Telescope Science Institute (STScI), which is operated by the Association of Universities for Research in Astronomy, Inc. under NASA contract NAS 5-26555.

\section{References}

DeLaney, T., Rudnick, L., Stage, M. D., et al. 2010, ApJ, 725, 2038

Finkelstein, S. L., Morse, J. A., Green, J. C., et al. 2006, ApJ, 641, 919

Hammer, N. J., Janka, H.-T., \& Müller, E. 2010, ApJ, 714, 1371

Margutti, R., Milisavljevic, D., Soderberg, A. M., et al. 2014, ApJ, 780, 21

Maeda, K., Kawabata, K., Mazzali, P. A., et al. 2008, Science, 319, 1220

Milisavljevic, D., Fesen, R. A., Chevalier, R. A., et al. 2012, ApJ, 751, 25

Milisavljevic, D. \& Fesen, R. A. 2015, Science, 347, 526

Ono, M., Nagataki, S., Ito, H., et al. 2013, ApJ, 773, 161

Pastorello, A., Cappellaro, E., Inserra, C., et al. 2013,ApJ, 767, 1

Smith, N. \& Arnett, W. D. 2014, ApJ, 785, 82 